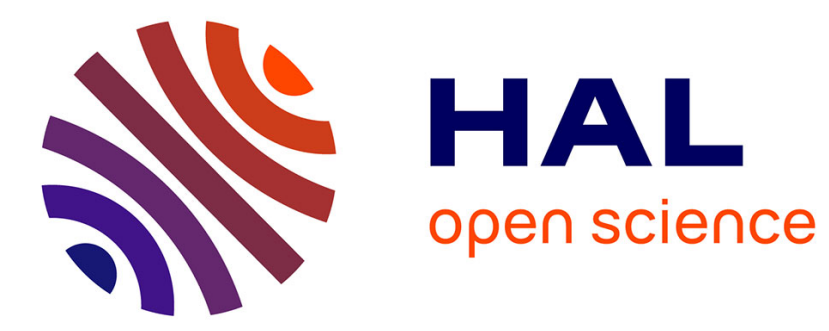

\title{
From EEG signals to brain connectivity: a model-based evaluation of interdependence measures.
}

Fabrice Wendling, Karim Ansari-Asl, Fabrice Bartolomei, Lotfi Senhadji

\section{To cite this version:}

Fabrice Wendling, Karim Ansari-Asl, Fabrice Bartolomei, Lotfi Senhadji. From EEG signals to brain connectivity: a model-based evaluation of interdependence measures.. Journal of Neuroscience Methods, 2009, 183 (1), pp.9-18. 10.1016/j.jneumeth.2009.04.021 . inserm-00387863

\section{HAL Id: inserm-00387863 https://www.hal.inserm.fr/inserm-00387863}

Submitted on 26 May 2009

HAL is a multi-disciplinary open access archive for the deposit and dissemination of scientific research documents, whether they are published or not. The documents may come from teaching and research institutions in France or abroad, or from public or private research centers.
L'archive ouverte pluridisciplinaire HAL, est destinée au dépôt et à la diffusion de documents scientifiques de niveau recherche, publiés ou non, émanant des établissements d'enseignement et de recherche français ou étrangers, des laboratoires publics ou privés. 


\title{
From EEG signals to brain connectivity: a model-based evaluation of interdependence measures
}

\author{
Fabrice Wendling ${ }^{1,2}$, Karim Ansari-Asl ${ }^{3}$, Fabrice Bartolomei ${ }^{4,5,6}$, Lotfi Senhadji ${ }^{1,2}$ \\ ${ }^{1}$ INSERM, U642, Rennes, F-35000, France \\ ${ }^{2}$ Université de Rennes 1, LTSI, F-35000, France \\ ${ }^{3}$ Shahid Chamran University, Faculty of Engineering, Ahvaz, Iran \\ ${ }^{4}$ INSERM, U751, Marseille, F-13000, France \\ ${ }^{5}$ AP-HM, Hôpital de la Timone, Service de Neurophysiologie Clinique, Marseille, F-13000, France \\ ${ }^{6}$ Aix Marseille Université, Faculté de Médecine, Marseille, F-13000, France \\ Corresponding author: fabrice.wendling@univ-rennes1.fr
}
Submitted to the Journal of Neuroscience Methods
Special issue on '(Multi-)frequency locking' March 2009

Revised version - April 2009 


\begin{abstract}
In the past, considerable effort has been devoted to the development of signal processing techniques aimed at characterizing brain connectivity from signals recorded from spatially-distributed regions during normal or pathological conditions. In this paper, three families of methods (linear and nonlinear regression, phase synchronization, and generalized synchronization) are reviewed. Their performances were evaluated according to a model-based methodology in which a priori knowledge about the underlying relationship between systems that generate output signals is available. This approach allowed us to relate the interdependence measures computed by connectivity methods to the actual values of the coupling parameter explicitly represented in various models of signal generation. Results showed that: (i) some of the methods were insensitive to the coupling parameter; (ii) results were dependent on signal properties (broad band versus narrow band); (iii) there was no "ideal" method, i.e. none of the methods performed better than the other ones in all studied situations. Nevertheless, regression methods showed sensitivity to the coupling parameter in all tested models with average or good performances. Therefore, it is advised to first apply these "robust" methods in order to characterize brain connectivity before using more sophisticated methods that require specific assumptions about the underlying model of relationship. In all cases, it is recommended to compare the results obtained from different connectivity methods to get more reliable interpretation of measured quantities with respect to underlying coupling. In addition, timefrequency methods are also recommended when coupling in specific frequency sub-bands ("frequency locking") is likely to occur as in epilepsy.
\end{abstract}

Keywords: connectivity; regression analysis; phase synchronization; generalized synchronization; model-based evaluation; EEG 


\section{Introduction}

It is commonly admitted that most of the brain functions are based on interactions between neuronal assemblies distributed within and across different cerebral regions. For instance, it has been shown that distant areas may activate in response to a particular cognitive task, the way coordination is achieved is not resolved yet (Uhlhaas and Singer 2006). In neurological disorders like epilepsy, it has also been shown that paroxysmal activity may involve networks extending over rather large regions (Bartolomei et al. 2001).

The identification of involved networks under either normal or pathological condition is still considered as a difficult and unsolved problem. Indeed, the characterization of the functional relationship between different brain areas from a measure of the statistical coupling between signals generated by these areas is not trivial, particularly because recording techniques only allow for indirect measurement of the activity in neuronal networks.

Over the past decades, methods aimed at estimating the functional connectivity between spatially-distributed regions from electrophysiological (scalp EEG, MEG or intracerebral EEG) data have received much attention. The consequence of this increasing interest is that now a plethora of methods for estimating functional connectivity is available, all based on different assumptions about the underlying model of relationship between analyzed signals. Therefore, many neuroscientists working in this field are confronted to this crucial question: "Given a particular context of study (cognitive or clinical research), which method should be used in order to best characterize the functional interactions between distant brain areas?"

The work presented in this paper stems from this question. Indeed, considering i) the large number of methods, ii) the possible dependence of results (provided by these methods) with respect to signal properties (stationary, linearity, bandwidth) and iii) the lack of reference regarding the underlying relationship between systems that generate analyzed signals, there is a need for performance comparison.

In this paper, we report a "model-based" evaluation of a number of "well-established" methods that have been used on electrophysiological data to assess brain connectivity. In order to restrict the scope of this study, we focused on bivariate methods in locally-stationary situations (thus providing frequency-independent measures). These methods were evaluated on the output signals of various models in which a coupling parameter can be tuned. Therefore, we were able to analyze the behavior of the methods with respect to changes of the coupling parameter and, using quantitative criteria, to compare their performances. 


\section{Background}

Since the middle of the last century, numerous techniques have been proposed for measuring the temporal evolution of the cross-correlation (in a wide sense) between signals recorded from spatially-distributed brain regions. Pioneer works started with the cross-correlation function (Barlow and Brazier 1954; Brazier and Casby 1952) in the time domain and with the coherence function (Brazier 1967; Storm van Leeuwen et al. 1976) in the frequency domain, just after fast Fourier transform (FFT) algorithms were introduced (Cooley and Tukey 1965). These methods were applied to the study of the propagation of inter-ictal events in human intracerebral EEG data (Brazier 1972). The averaged coherence was also used on signals acquired from both hemispheres in order to study the evolution of inter-hemispheric interactions on the whole duration of partial seizures (Gotman 1987). At the same time, this method revealed the existence of activities that could propagate over short-range or longrange connection fibers (Thatcher et al. 1986). Later, the averaged coherence also revealed possible synchronization mechanisms occurring at the onset of seizures (Duckrow and Spencer 1992). In this context, a frequentlyaddressed question was also the estimation of time delays (Gotman 1983; Ktonas and Mallart 1991) as a measure of the "latency" which could provide insights into propagation phenomena between distant structures. A variant of the classical computation of the coherence function is to use of time-varying linear models (autoregressive models, multiple windowing) in order to estimate cross- and auto-spectra. Some studies reported results from these parametric methods and showed their potential value for measuring the degree of synchronization of interictal and ictal EEG signals and for characterizing the relationship between brain oscillations in the time and/or frequency domain (Franaszczuk and Bergey 1999; Haykin et al. 1996). Later, complementary approaches were developed to estimate the direction of coupling between signals while also taking into account the possible influence of external sources (see review in (Gourevitch et al. 2006)).

The aforementioned methods are said to be linear. This means that the estimator that is used or the model that is assumed for signals can only capture the linear properties of the relationship between time series. However, most of the mechanisms at the origin of EEG signals are probably nonlinear. Starting from this fact, numerous studies were devoted to the development of nonlinear methods (Pikovsky et al. 2001). A first family based on mutual information (Mars and Lopes da Silva 1983) or on nonlinear regression (Pijn and Lopes da silva 1993; Wendling et al. 2001b) was introduced in the field of EEG about twenty years ago. A second family developed later on, based on tools already available in the field of nonlinear dynamical systems and chaos (Iasemidis 2003; Lehnertz 1999). This second family can be divided into two groups: phase synchronization (PS) methods (Bhattacharya 2001; Rosenblum et al. 2004) and generalized synchronization (GS) methods (Arnhold et al. 1999; Stam and van 
Dijk 2002). PS methods estimate the instantaneous phase of each signal and then compute a quantity based on co-variation of extracted phases to determine the degree of relationship. GS methods also consist of two steps: the reconstruction of state space trajectories from time series signals and the computation of a similarity index on reconstructed trajectories.

\section{Evaluated methods}

The above brief literature review shows that the number of developed methods and variants is quite large. In this paper, we focus on 10 methods that have been applied to electro- or magneto- encephalographic signals (EEG, depth-EEG or MEG) in numerous studies. These 10 methods can be grouped into three main families: (1) regression methods: Pearson correlation coefficient $\left(\mathrm{R}^{2}\right)$, coherence function $(\mathrm{CF})$ and nonlinear correlation coefficient $\left(h^{2}\right)$; (2) phase synchronization methods: Hilbert phase entropy (HE), Hilbert mean phase coherence (HR), wavelet phase entropy (WE) and wavelet mean phase coherence (WR); (3) generalized synchronization methods: two similarity indexes (S, N) and a synchronization likelihood (SL). Main theoretical aspects are summarized hereafter.

\subsection{Regression methods: $R^{2}, C F$ and $h^{2}$}

For two time series $x(t)$ and $y(t)$, the Pearson correlation $R^{2}$ coefficient is defined as

$$
R^{2}=\max _{\tau} \frac{\operatorname{cov}^{2}(x(t), y(t+\tau))}{\operatorname{var}(x(t)) \cdot \operatorname{var}(y(t+\tau))}
$$

where var, cov, and $\tau$ denote respectively variance, covariance, and time shift.

The magnitude-squared coherence function (CF) can be formulated as (Bendat and Piersol 1971):

$\left|\rho_{x y}(f)\right|^{2}=\frac{\left|S_{x y}(f)\right|^{2}}{S_{x x}(f) \cdot S_{y y}(f)}$

where $S_{x x}(f)$ and $S_{y y}(f)$ respectively denote the power spectral densities of $x(t)$ and $y(t)$, and where $S_{x y}(f)$ denotes their cross-spectral density. It is the counterpart of the $R^{2}$ coefficient in the frequency domain. It 
can be interpreted as the squared modulus of a frequency-dependent complex correlation coefficient. Regarding the nonlinear regression analysis, we implemented the so-called $h^{2}$ method. This method was introduced in the field of EEG analysis by Lopes da Silva and colleagues (Lopes da Silva et al. 1989). It was also evaluated in several studies (Ansari-Asl et al. 2006; Wendling et al. 2001a) using coupled oscillators. Main theoretical aspects regarding this approach were revisited in (Kalitzin et al. 2007). In brief, in this method, a nonlinear correlation coefficient referred to as $h^{2}$ is computed based on the fitting of a nonlinear curve $g($.$) which approximates$ the statistical relationship between $x(t)$ and $y(t)$ :

$h_{x y}^{2}=\max _{\tau}\left(1-\frac{\operatorname{var}(y(t+\tau) / x(t))}{\operatorname{var}(y(t+\tau))}\right)$ where $\operatorname{var}(y(t+\tau) / x(t)) \triangleq \underset{g}{\arg \min }\left(E[y(t+\tau)-g(x(t))]^{2}\right)$

In practice, function $g($.$) can be obtained from the piece-wise linear approximation between the samples of the$ two time series $x(t)$ and $y(t)$ (Pijn 1990).

\subsection{Phase synchronization methods: $H E, H R, W E, W R$}

The first step for estimating the phase synchronization is to extract the instantaneous phase of each signal (Rosenblum et al. 2004). Two different techniques are considered in this paper: the Hilbert transform and the wavelet transform. The second step is the definition of an appropriate index to measure the degree of synchronization between estimated instantaneous phases.

The Hilbert transform is used to determine the analytical signal associated to a real time series $x(t)$ :

$Z_{x}(t)=x(t)+i H[x(t)]=A_{x}^{H}(t) e^{i \phi_{x}^{H}(t)}$

where $H, \phi_{x}^{H}$, and $A_{x}^{H}(t)$ are respectively the Hilbert transform, the phase, and the amplitude of $x(t)$.

The complex continuous wavelet transform can also be used for estimating the phase of a signal (Delprat et al. 1992; Le Van Quyen et al. 2001; Senhadji et al. 1996):

$W_{x}(t)=(\psi * x)(t)=\int \psi\left(t^{\prime}\right) x\left(t-t^{\prime}\right) d t^{\prime}=A_{x}^{W}(t) \cdot e^{i \phi_{x}^{W}(t)}$ 
where $\psi, \phi_{x}^{W}$, and $A_{x}^{W}(t)$ are respectively a wavelet function (e.g., Morlet used here), the phase, and the amplitude of $x(t)$. Once the phase estimation has been performed on two considered signals, a synchronization index can be defined to quantify the phase relationship. In this paper, we present two different indexes, both based on the shape of the probability density function (pdf) of the modulo $2 \pi$ phase difference $\left(\phi=\left(\phi_{x}-\phi_{y}\right) \bmod 2 \pi\right)$.

The first index is devised from the Shannon entropy and defined by $\rho=1+\frac{1}{\ln (M)} \sum_{i=1}^{M} p_{i} \ln p_{i} \quad$ where $M$ is the number of bins used to obtain the pdf, $p_{i}$ is the probability of finding the phase difference $\phi$ within the $i^{\text {th }}$ bin (Munari et al. 1994). The second index, refered to as the "mean phase coherence", is given by $\left|\mathbb{E}\left[e^{i \phi}\right]\right|$. As described in (Mormann et al. 2000), it can be estimated by: $R=\left|\frac{1}{N} \sum_{t=0}^{N-1} e^{i \phi(t)}\right|$ where $N$ is the length of the two time series.

Combining the two phase estimators $(\mathrm{H}, \mathrm{W})$ and the two synchronization indexes $(\mathrm{E}, \mathrm{R})$, we considered four different measures of interdependencies, denoted as Hilbert entropy (HE), Hilbert mean phase coherence (HR), wavelet entropy (WE) and wavelet mean phase coherence (WR).

\subsection{Generalized synchronization based methods: $S, N, S L$}

Generalized synchronization approaches were introduced to investigate the interactions between nonlinear dynamical systems without any knowledge about the governing equations. They generally proceed according to two steps. First, a state space trajectory is reconstructed from each scalar time series using a time delay embedding method (Takens 1981). For each discrete time $n$ a delay vector corresponding to a point in the state space reconstructed from $x$ is defined as:

$X_{n}=\left(x_{n}, x_{n+\tau}, \ldots, x_{n+(m-1) \tau}\right) ; n=1, \ldots, N$

where $m$ is the embedding dimension and $\tau$ denotes time lag. The state space trajectory of $y$ is reconstructed in the same way. Second, a synchronization degree is determined using a suitable measure. Three measures, all based on conditional neighborhood, are presented in this section. The general principle is to quantify the proximity, in the second state space, of the points whose temporal indices correspond to neighbor points in the first 
state space. Two of these measures $S$ and $N$ (Arnhold et al. 1999), which are also sensitive to the direction of interaction, originate from this principle. They are based on an Euclidean distance:

$S^{(k)}(X \mid Y)=\frac{1}{N} \sum_{n=1}^{N} \frac{R_{n}^{(k)}(X)}{R_{n}^{(k)}(X \mid Y)}$

$N^{(k)}(X \mid Y)=\frac{1}{N} \sum_{n=1}^{N} \frac{R_{n}^{(N-1)}(X)-R_{n}^{(k)}(X \mid Y)}{R_{n}^{(N-1)}(X)}$

with $R_{n}^{(k)}(X)=\frac{1}{k} \sum_{j=1}^{k}\left(X_{n}-X_{r_{n, j}}\right)^{2}$ and $R_{n}^{(k)}(X \mid Y)=\frac{1}{k} \sum_{j=1}^{k}\left(X_{n}-X_{s_{n, j}}\right)^{2}$

where $r_{n, j}, j=1, \ldots, k$ and $s_{n, j}, j=1, \ldots, k$ respectively stand for the time indices of the $k$ nearest neighbors of $X_{n}$ and $Y_{n}$.

It is noteworthy that the third measure, referred to as the synchronization likelihood (SL) (Stam and van Dijk 2002), is a measure of multivariate synchronization. Here, for simplicity, we only consider the bivariate case. The estimated probability that embedded vectors $X_{n}$ are closer to each other than a distance $\varepsilon$ is:

$$
P_{x, n}^{\varepsilon}=\frac{1}{2\left(w_{2}-w_{1}\right)} \sum_{\substack{j=1 \\ w_{1} \backslash\left\langle n-j|j| w_{2}\right.}}^{N} \theta\left(\varepsilon-\left|X_{n}-X_{j}\right|\right)
$$

where $|\cdot|$ is the Euclidean distance; $\theta$ stands for the Heaviside step function, $w_{1}$ is the Theiler correction and $w_{2}$ determines the length of sliding window. Letting $P_{x, n}^{\varepsilon}=P_{y, n}^{\varepsilon}=P_{r e f}$ be a small arbitrary probability, the above equation for $X_{n}$ and its analogous for $Y_{n}$, gives the critical distances $\varepsilon_{x, n}$ and $\varepsilon_{y, n}$ from which we can determine if simultaneously $X_{n}$ is close to $X_{j}$ and $Y_{n}$ is close to $Y_{j}$, i.e., $H_{n, j}=2$ in the equation below

$$
H_{n, j}=\theta\left(\varepsilon_{x, n}-\left|X_{n}-X_{j}\right|\right)+\theta\left(\varepsilon_{y, n}-\left|Y_{n}-Y_{j}\right|\right)
$$

Synchronization likelihood at time $n$ can be obtained by averaging over all values of $j$

$$
S L_{n}=\frac{1}{2 P_{r e f}\left(w_{2}-w_{1}\right)} \sum_{\substack{j=1 \\ w_{1}<n-j \mid<w_{2}}}^{N}\left(H_{n, j}-1\right)
$$


All aforementioned measures are normalized between 0 and 1; the 0 value means that the two signals are completely independent. On the opposite, the 1 value means that the two signals are completely synchronized.

Finally, in order to deal with the evolution, in time, of brain connectivity the three measures described above can be estimated over a sliding window.

\section{Model-based evaluation methodology}

In order to perform an objective comparison of methods, we propose a model-based approach in which a priori knowledge about the underlying relationship between systems that generate output signals is available. As illustrated in figure 1, connectivity methods are applied on the output signals $X$ and $Y$ produced by two models of signal generation $S_{1}$ and $S_{2}$. The coupling between these two models can be adjusted using a coupling parameter $C$. Some noise $\left(N_{l}\right.$ and $\left.N_{2}\right)$ can be added to output signals. Evaluated methods provide a quantity $Q$ (normalized between 0 and 1) measured from $X$ and $Y$ and supposed to characterize the connectivity between the two models. Therefore, this framework offers the possibility to study the behavior of candidate connectivity methods with respect i) to the coupling parameter $C$, ii) to the type of model that is used to generate $X$ and $Y$ and iii) to the features of noise present on output signals. In this context, the "ideal" method would provide a quantity $Q$ which would reflect any variation of coupling parameter $C$ for any model of signal generation, with low bias and variance and high sensitivity. This consideration led us to propose different models for generating outputs signals (section 4.1) and three criteria (section 4.2) to evaluate the behavior of connectivity methods with respect to the variation of parameter $\mathrm{C}$.

\subsection{Models of signal generation}

Table 1 gives an overview of signal generation models used in this study. As depicted, these models can be divided into three main families: i) models of coupled stochastic signals (M1 and M2), ii) models of coupled nonlinear dynamical systems (M3 and M4) and iii) models of coupled neuronal populations (M5). In each family, various situations were considered in order to check for the influence of signal properties (narrow versus broad band activity) or the influence of noise on connectivity measures. The main characteristics of each model are described hereafter.

\subsubsection{Coupled broad band signals: $M 1$}


Model $\mathrm{M}_{1}$ generates two broad band signals $\left(x_{1}, x_{2}\right)$ built using two independent and a common white noises respectively $\left(N_{l}, N_{2}\right)$ and (N3):

$$
\begin{aligned}
& x_{1}=(1-C) N_{1}+C N_{3} \\
& x_{2}=(1-C) N_{2}+C N_{3}
\end{aligned}
$$

where $0 \leq C \leq 1$ is the coupling degree; for $C=0$ the signals are independent and for $C=1$ they are identical.

\subsubsection{Coupled narrow band signals: M2(PR) and M2(AR)}

In $\mathrm{M}_{2}$, two narrowband signals around a frequency $f_{0}$ are generated from four lowpass filtered white noises $\left(N F_{1}, N F_{2}, N F_{3}\right.$, and $\left.N F_{4}\right)$. They are combined in two ways in order to share either a phase relationship (PR) or an amplitude relationship (AR), only:

$$
\begin{aligned}
& P R:\left\{\begin{array}{l}
x_{1}=A_{1} \cos \left(2 \pi f_{0} t+\phi_{1}\right) \\
x_{2}=A_{2} \cos \left(2 \pi f_{0} t+C \phi_{1}+(1-C) \phi_{2}\right)
\end{array}\right. \\
& A R:\left\{\begin{array}{l}
x_{1}=A_{1} \cos \left(2 \pi f_{0} t+\phi_{1}\right) \\
x_{2}=\left(C A_{1}+(1-C) A_{2}\right) \cos \left(2 \pi f_{0} t+\phi_{2}\right)
\end{array}\right.
\end{aligned}
$$

where $A_{1}=\sqrt{N F_{1}^{2}+N F_{2}^{2}}, A_{2}=\sqrt{N F_{3}^{2}+N F_{4}^{2}}, \phi_{1}=\arctan \left(\frac{N F_{2}}{N F_{1}}\right), \phi_{2}=\arctan \left(\frac{N F_{4}}{N F_{3}}\right)$, and $0 \leq C \leq 1$. For $C=0$ the two signals have independent phase and amplitude and for $C=1$ they have identical phase or amplitude.

We also used some nonlinear deterministic systems for modeling nonlinear synchronized coupled oscillators. Here we report results obtained for two of them: Rössler-Rössler coupled system ( $\left.\mathrm{M}_{3}\right)$ (Pikovsky et al. 1996) and Hénon-Hénon coupled system $\left(\mathrm{M}_{4}\right)$ (Bhattacharya 2001)

\subsubsection{Coupled Rössler systems: M3}

In $\mathrm{M}_{3}$ two Rössler systems (Rossler 1976) are coupled and the driver system is given by:

$$
\begin{aligned}
& \frac{d x_{1}}{d t}=-\omega_{x} x_{2}-x_{3} \\
& \frac{d x_{2}}{d t}=\omega_{x} x_{1}+0.15 x_{2} \\
& \frac{d x_{3}}{d t}=0.2+x_{3}\left(x_{1}-10\right)
\end{aligned}
$$


and the response system is described by:

$$
\begin{aligned}
& \frac{d y_{1}}{d t}=-\omega_{y} y_{2}-y_{3}+C\left(x_{1}-y_{1}\right) \\
& \frac{d y_{2}}{d t}=\omega_{y} y_{1}+0.15 y_{2} \\
& \frac{d y_{3}}{d t}=0.2+y_{3}\left(y_{1}-10\right)
\end{aligned}
$$

Here $\omega_{x}=0.95, \omega_{y}=1.05$, and $C$ is the coupling degree.

\subsubsection{Coupled Hénon systems: M4a and M4b}

Hénon map (Hénon 1976) is a nonlinear deterministic which is discrete by construction. In model $\mathrm{M}_{4}$, we make use of two of them to simulate a unidirectional coupled system. The driver system is given by:

$$
x[n+1]=1.4-x^{2}[n]+b_{x} x[n-1]
$$

and the response system is

$$
y[n+1]=1.4-\left(c x[n] y[n]+(1-c) y^{2}[n]\right)+b_{y} y[n-1]
$$

where $C$ is a coupling degree and $b_{x}=0.3$; to create different situation, once $b_{y}$ is set to 0.3 to have two identical systems $\left(\mathrm{M}_{4 \mathrm{a}}\right)$ and once $b_{y}$ is set to 0.1 to have two non-identical systems $\left(\mathrm{M}_{4 \mathrm{~b}}\right)$.

\subsubsection{Coupled neuronal populations: M5}

In order to simulate realistic temporal dynamics encountered in real depth-EEG signals, as recorded in patients with drug-resistant epilepsy during pre-surgical evaluation, a physiologically-relevant computational model of EEG generation was used. As illustrated in figure 2, this model represents the activity of two distant - and possibly coupled - neuronal populations. Each population generates a local field potential $(X$ and $Y)$ that can be seen as a depth-EEG signal if one does not consider the source-electrode transfer function. Readers may refer to (Wendling et al. 2000) for detailed description and generalization to more than 2 populations. In this model, each population contains two subpopulations of neurons that mutually interact via excitatory or inhibitory feedback: main pyramidal cells and local interneurons. The excitatory influence from non-specific afferents is modeled by a Gaussian input noise ( $N_{1}$ or $N_{2}$ ) that globally represents the average density of afferent action potentials on population 1 and population 2. Pyramidal cells are excitatory neurons that project their axons to distant areas of 
the brain. The model accounts for this type of connection by using the average pulse density of action potentials from the main cells of population 1 as an excitatory input to population 2. In addition, this uni-directional connection from population 1 to population 2 is characterized by parameter $C$ which represents the "synaptic gain", allowing for tuning the degree of coupling between the two populations. Other parameters include excitatory and inhibitory gains in feedback loops as well as average number of synaptic contacts between subpopulations. The model was used to generate two kinds of signals: background (BKG) and spiking (SPK) activity respectively obtained for normal and increased excitation/inhibition ratio in each population. For both cases, bivariate data were simulated. The normalized coupling parameter $C$ was varied from 0 (independence between signals) to 1 (dependence between signal, similar temporal dynamics).

\subsection{Comparison criteria}

Three criteria were defined in order to quantitatively evaluate the behavior of the methods presented in section 3 on time-series simulated from the coupled models presented in section 4.1 for different values of the coupling parameter $C$. The two first criteria are classical:

- the mean square error (MSE) under null hypothesis (i.e., independence between two signals) can be interpreted as a quadratic bias, defined by $\mathbb{E}\left[(\hat{Q}-Q)^{2}\right]$ where $E$ is the mathematical expectation, $Q=0$ (no coupling) and $\hat{Q}$ is the estimation of $Q$,

- the mean variance (MV) computed over all values $C=c_{i}, i=1,2, \ldots, I$ of the degree of coupling and defined as $\frac{1}{I} \sum_{i=1}^{I} \mathbb{E}\left[\left(\hat{Q}_{i}-\mathbb{E}\left[Q_{i}\right]\right)^{2}\right]$ where $I$ is number of coupling degree points and $\hat{Q}_{i}$ is the estimated relationship for the coupling degree $c_{i}$.

The third performance criterion, referred to as the median of local relative sensitivity (MLRS), is novel. It was introduced to quantify the sensitivity of the method with respect to changes in the coupling degree. The MLRS is given by:

$$
\operatorname{MLRS}=\operatorname{Median}\left(S_{i} / \bar{\sigma}_{i}\right), \quad S_{i}=\frac{\hat{Q}_{i+1}-\hat{Q}_{i}}{c_{i+1}-c_{i}}, \quad \bar{\sigma}_{i}=\sqrt{\frac{\hat{\sigma}_{i}^{2}+\hat{\sigma}_{i+1}^{2}}{2}}
$$


where $S_{i}$ is the increase rate of the estimated relationship and $\bar{\sigma}_{i}$ is the square root of the average of estimated variances associated to two adjacent values of the coupling degree. One can also use the median of the distribution of local relative sensitivity instead of its mean because the fluctuation in its estimation may make this distribution very skewed.

Methods with lower values of MSE and MV can be considered to have better performances. Conversely, higher MLRS values indicate better performances.

\section{Results}

For all models of signal generation (M1-M5) and for all values of parameter $C$ (from 0 to 1 by steps of 0.1 ), Monte Carlo simulations were performed in order to assess statistical properties of connectivity measures provided by methods described in section 3 and to comparatively evaluate their performances according to criteria presented in section 4.2. To proceed, long duration time-series signals (200000 samples) sampled at $256 \mathrm{~Hz}$ were generated using each model, for discrete values of the coupling parameter $\mathrm{C}$, ranging from 0 to 1 , by steps of 0.1 . All quantities were estimated over a sliding window of 512 samples ( 2 seconds of activity) moving by steps of 64 samples (75\% overlapping) and then averaged over time. In GS methods, parameter $\tau$ was estimated as follows. First the mutual information as a function of positive time lag was plotted and then, as described in (Sills et al. 2000), time lag $\tau$ was chosen as the abscissa value corresponding to the first minimum this curve. The embedded dimension $m$, in this family of methods, was determined from the Cao method (Cao 1997). To limit the length of this section, we will only detail the results obtained in two cases (models M2(PR) and M5 (SPK)) and provide a global synthesis that summarizes the performances of the three families of methods in all considered situations.

Results obtained in model $\mathrm{M}_{2}$ for phase relationship only (PR), are shown in figure 3. They show that phase synchronization methods (HE, HR, WE, WR) exhibited higher performances than other methods, as it could be expected. Similarly, results obtained with $R^{2}$ and $h^{2}$ methods were correct. On the opposite, generalized synchronization methods and coherence (CF) had lower performances (S, SL: low sensitivity to $C$ variation, S: strong bias, SL: strong variance, CF: low sensitivity). Regarding the variance of estimation, it can be noticed that some methods $\left(\mathrm{R}^{2}, \mathrm{WE}\right)$ perform better than others. For higher coupling values, the variance decreases for all methods, except SL. 
Figure 4 shows the signals generated by the neuronal population model (M5) in the case of spiking (SPK) activity. The temporal dynamics of these signals reproduce those often observed during epileptic seizures. It is noteworthy that similar signals were used in a previous attempt for qualitatively comparing different correlation estimators (Quian Quiroga et al. 2002). As depicted, most of the methods could detect the increase of the coupling parameter in the model. Some methods exhibited strong bias (HE). As an interesting result, it could be observed that WE and CF were almost blind to the increase of coupling. Similarly, HE and WR only displayed small increase with increasing of degree of coupling but their variance was low. $\mathrm{R}^{2}, \mathrm{~h}^{2}, \mathrm{~S}$ and HR methods exhibited good sensitivity. However, MSE under null hypothesis was found to be high for HR. Globally, the variance was found to decrease with the coupling parameter all methods, except CF.

The two examples described above show that the behavior can strongly vary from one method to another within the same situation. In order to make a global comparison of the 10 studied methods, we computed the MSE under null hypothesis, the MV and the MRLS (both averaged over the coupling values) for all situations. Results are summarized in table 2. From this table, it can be observed that the $\mathrm{R}^{2}$ method exhibited good results in model $\mathrm{M} 1$ and $\mathrm{M} 5(\mathrm{BKG})$ and that the $\mathrm{h}^{2}$ method showed good sensitivity to coupling changes in model M5 (SPK). Among regression methods, the average coherence $\mathrm{CF}$ showed poorer performances. Among phase synchronization methods, the wavelet entropy (WE) performed better than other methods of the same family, in particular in model M2(PR). It is noteworthy that the four phase synchronization methods were not able to detect coupling changes in model M2(AR). Regarding generalized synchronization methods, results showed that best performances are obtained in models of coupled nonlinear systems. In particular, the best results were obtained with the similarity index $\mathrm{S}$ in model M4b.

We also averaged the results obtained in each family of methods (regression, PS or GS). Results are synthesized in figure 5. On one hand, a criterion-by-criterion analysis leads to the following remarks. Regarding the sensitivity to the coupling parameter (MRLS, figure 5-a), regression methods $\left(\mathrm{R}^{2}, \mathrm{~h}^{2}\right.$ and $\mathrm{CF}$ ) and PS methods (HE, HR, WE, WR) perform better than GS (S, N, SL) methods in model M1. As it could be expected, PS methods showed no sensitivity to coupling in model M2(AR) and highest sensitivity in model M2(PR). Globally GS methods showed good sensitivity to coupling in model M4 (Hénon) but were outperformed by PS methods in model M3 (Rössler). Regression methods $\left(\mathrm{R}^{2}, \mathrm{~h}^{2}\right.$ but not $\left.\mathrm{CF}\right)$, showed good sensitivity to the coupling parameter in the model of coupled neuronal populations. Regarding the mean variance (MV, figure 5-b), regression methods $\left(\mathrm{R}^{2}, \mathrm{~h}^{2}\right)$ displayed low values in model M1. In models M2 (PR), quasi-similar variance values were obtained for the three families. In models M2 (AR), lower variance was obtained for GS methods still keeping some sen- 
sitivity to the coupling parameter. PS methods showed very low variance in model M3 (Rössler). In model M4 (Hénon), GS methods exhibited the highest variance but were, in turn, the most sensitive to the coupling parameter. For model M5, PS and regression methods performed better than GS methods. Regarding the mean square error under null hypothesis (MSE, figure 5-c), results showed that some methods can be characterized by a high bias value like GS methods in model M2, PS methods in model M3 and GS methods in model M5.

On the other hand, a model-by-model analysis of figure 5 shows that for model $\mathrm{M}_{1}$ regression methods perform better than others as the MV is the lowest while the MRLS is the highest. For model $\mathrm{M}_{2}$ (in the case of PR), it is evident that PS methods are the most appropriate. For model $\mathrm{M}_{2}$ (in the case of AR), there is no consensus for the best method. For model $\mathrm{M}_{3}$, PS methods outperform others although they are characterized by higher MSE values. For model $\mathrm{M}_{4}$ (considering the four situations), GS methods have the lowest MSE and PS methods have the lowest MV. As far as the MRLS is concerned, these two groups of methods perform equally. Finally, for the neuronal population model $\mathrm{M}_{5}$, regression methods outperform others in the case of normal background EEG activity. For spiking epileptic-like activity, these methods, in addition to PS methods have also higher performances than GS methods.

\section{Discussion}

\section{Relevance in the field of brain research and progress with respect to already published studies}

Numerous methods have been introduced to tackle the difficult problem of characterizing the statistical relationship between signals without a priori knowledge about the nature of this relationship. In brain research, a number of methods have been proposed and/or used to study normal or pathological processes. Such methods play a key role as they are supposed to provide important information regarding brain connectivity from electrophysiological recordings.

During the ten past years, some efforts have been made for comparing methods but mainly qualitatively (David et al. 2004; Quian Quiroga et al. 2002) and for particular applications (Mormann et al. 2005; Pereda et al. 2005). In this paper, we presented three families of methods (regression, phase synchrony and general synchronization) and we compared their performances on the basis of simulations produced by various models (including a neurophysiologically-relevant one) in which a parameter can be tuned in order to adjust the coupling between two "systems" from which output signals are generated. Through this model, we were able to study the relationship between the coupling parameter in the models and the quantity actually measured on output signals using "connectivity" methods. 
In this regard, this approach differs from that of Schiff et al. (Schiff et al. 1996) who evaluated one method to characterize dynamical interdependence (based on mutual nonlinear prediction) on both simulated (coupled identical and non identical chaotic systems) and real (activity of motoneurons within a spinal cord motoneuron pool) data. It also differs from other evaluation studies which mainly focused on qualitative comparisons (David et al. 2004; Quian Quiroga et al. 2002) and for specific applications (Mormann et al. 2005; Pereda et al. 2005). In the particular field of EEG analysis, the model of coupled neuronal populations is of particular relevance since it generates realistic temporal dynamics. In this model, for background activity (that can be considered as a broadband random signal), we found that coherence and phase synchrony methods (except HR) were not sensitive to the increase of the coupling parameter whereas regression methods (linear and nonlinear) exhibited better sensitivity. This result may be explained by the fact that the interdependence between simulated signals is not entirely determined by a phase relationship. This point is crucial since it illustrates the fact that the choice of the method used to characterize the relationship between signals is critical and may lead to possible incorrect interpretation of results obtained on EEG data.

In addition, as background activity can be recorded in epileptic patients during interictal periods, our results also relate to those recently published by Morman et al. (Mormann et al. 2005) in the context of seizure prediction. For thirty different measures obtained from univariate and bivariate approaches, authors evaluated their ability to distinguish between the interictal period and the pre-seizure period (sensitivity and specificity of all measures were compared using receiver-operating-characteristics). In both types of approach (and consequently for bivariate methods similar to those implemented in the present study) they also found that linear methods performed equally good or even better than nonlinear methods.

\section{Limitations of this study}

In this report, results about the characterization of the direction of coupling were not dealt with. This difficult issue has already been addressed in various reports. For instance, Quian Quiroga et al. (Quian Quiroga et al. 2002) quantitatively tested two interdependence measures on coupled nonlinear oscillators for their ability to determine whether one the two systems drives the other. Other families of approaches where also developed to estimate directional properties of the relationship between signals while taking into account possible influences of external sources (Gourevitch et al. 2006). According to these approaches, causality between two signals (in Granger's sense) is estimated based on the predictability of one signal from the immediate past of the other signal. 
Besides, the methods evaluated in this study are independent from frequency. However, in some situation, it might be crucial to take frequency into account. Indeed, frequency-independent methods may not be able to reveal some phenomena like a hypersynchronization in a narrow frequency band as sometimes observed at the onset of partial seizures. An example is illustrated in figure 6 which displays the depth-EEG signals recorded from mesial temporal lobe structures in a patient candidate to surgery. As observed, the seizure onset is marked by the appearance of a fast activity clearly localized in the time-frequency representations (figure 6-b) of the two depthEEG signals (figure 6-a). A "frequency-locking" phenomenon is revealed by the computation of the linear correlation as the function of time and frequency (see methods in (Ansari-Asl et al. 2005)). As shown in figure 6-c, at seizure onset, the two depth-EEG signals are correlated in a well-defined frequency sub-band (around $30 \mathrm{~Hz}$ ). More importantly, it should be noticed that time-independent methods $\left(\mathrm{R}^{2}\right.$ and $\left.\mathrm{h}^{2}\right)$ that showed relatively robust in simulations do not show significant changes at seizure onset (figure 6-d and 6-e). As these methods apply on broadband signals, they are unable to detect the increase of correlation occurring at a specific frequency.

\section{Summary of the main findings}

The main findings of this study can be summarized as follows: $(i)$ some of the compared methods are insensitive to the coupling parameter in the model; (ii) results are dependent on signal properties (broad band versus narrow band); (iii) generally speaking, there is no universal method to deal with signal coupling, i.e., none of the studied methods performed better than the other ones in all studied situations.

Nevertheless, we notice that simple methods like $\mathrm{R}^{2}$ and $\mathrm{h}^{2}$ methods showed to be sensitive to the coupling parameter in the model with average or good performances. Therefore, it might be reasonable to first apply these "robust" regression methods in order to characterize brain connectivity before using more sophisticated methods that require specific assumptions about the underlying model of relationship. In all cases, it is highly recommended to compare the results obtained from different connectivity methods to get more reliable interpretation of measured quantities with respect to underlying coupling. Following this idea, results suggest that some information could be inferred about the nature of the relationship based on the comparison of indexes provided by the different methods although the question of the physiological plausibility of the nature of the relationship will always remain.

In addition, some coupling phenomena might correspond to increased correlation between output signals, but in one or several specific sub-band(s). In such cases, time-frequency methods may also be of help. In this category, it should be reminded that a characterization of the relationship based on a "same frequency to same frequency" approach can only reveal the linear component of this relationship. A more general approach would allow for the 
computation of the correlation (in a wide sense) between signals filtered in different frequency sub-bands but might also lead to additional difficulties in the interpretation of results.

\section{References}

Ansari-Asl, K., Bellanger, J. J., Bartolomei, F., Wendling, F., and Senhadji, L. (2005). "Time-frequency characterization of interdependencies in nonstationary signals: application to epileptic EEG." IEEE Trans Biomed Eng, 52(7), 1218-26.

Ansari-Asl, K., Senhadji, L., Bellanger, J. J., and Wendling, F. (2006). "Quantitative evaluation of linear and nonlinear methods characterizing interdependencies between brain signals." Phys Rev E Stat Nonlin Soft Matter Phys, 74(3 Pt 1), 031916.

Arnhold, J., Grassberger, P., Lehnertz, K., and Elger, C. E. (1999). "A robust method for detecting interdependences: application to intracranially recorded EEG." Physica D: Nonlinear Phenomena, 134(4), 419-430.

Barlow, J. S., and Brazier, M. A. (1954). "A note on a correlator for electroencephalographic work." Electroencephalogr Clin Neurophysiol, 6(2), 321-5.

Bartolomei, F., Wendling, F., Bellanger, J. J., Regis, J., and Chauvel, P. (2001). "Neural networks involving the medial temporal structures in temporal lobe epilepsy." Clin Neurophysiol, 112(9), 1746-60.

Bendat, J., and Piersol, A. (1971). Random data: analysis and measurement procedures, Willey-Interscience.

Bhattacharya, J. (2001). "Reduced degree of long-range phase synchrony in pathological human brain." Acta Neurobiol Exp (Wars), 61(4), 309-18.

Brazier, M. A. (1967). "Varieties of computer analysis of electrophysiological potentials." Electroencephalogr Clin Neurophysiol, Suppl 26:1-8.

Brazier, M. A. (1972). "Spread of seizure discharges in epilepsy: anatomical and electrophysiological considerations." Exp Neurol, 36(2), 263-72.

Brazier, M. A., and Casby, J. U. (1952). "Cross-correlation and autocorrelation studies of electroencephalographic potentials." Electroencephalogr Clin Neurophysiol, 4(2), 201-11.

Cao, L. (1997). "Practical method for determining the minimum embedding dimension of a scalar time series." Physica D: Nonlinear Phenomena, 110(1-2), 43-50.

Cooley, J. W., and Tukey, J. W. (1965). "An Algorithm for the Machine Calculation of Complex Fourier Series." Math. Comput., 19, 297-301.

David, O., Cosmelli, D., and Friston, K. J. (2004). "Evaluation of different measures of functional connectivity using a neural mass model." Neuroimage, 21(2), 659-73.

Delprat, N., Escudie, B., Guillemain, P., Kronland-Martinet, R., Tchamitchian, P., and Torresani, B. (1992). "Asymptotic wavelet and Gabor analysis: extraction of instantaneous frequencies." Information Theory, IEEE Transactions on, 38(2), 644-664.

Duckrow, R. B., and Spencer, S. S. (1992). "Regional coherence and the transfer of ictal activity during seizure onset in the medial temporal lobe." Electroencephalogr Clin Neurophysiol, 82(6), 415-22.

Franaszczuk, P. J., and Bergey, G. K. (1999). "An autoregressive method for the measurement of synchronization of interictal and ictal EEG signals." Biol Cybern, 81(1), 3-9.

Gotman, J. (1983). "Measurement of small time differences between EEG channels: method and application to epileptic seizure propagation." Electroencephalogr Clin Neurophysiol, 56(5), 501-14.

Gotman, J. (1987). "Interhemispheric interactions in seizures of focal onset: data from human intracranial recordings." Electroenceph. Clin. Neurophysiol, 67, 120-133.

Gourevitch, B., Bouquin-Jeannes, R. L., and Faucon, G. (2006). "Linear and nonlinear causality between signals: methods, examples and neurophysiological applications." Biol Cybern, 95(4), 349-69.

Haykin, S., Racine, R. J., Xu, Y., and Chapman, C. A. (1996). "Monitoring neural oscillation and signal transmission between cortical regions using time-frequency analysis of electroencephalographic activity." Proceedings of IEEE, 84, 1295-1301.

Hénon, M. (1976). "A two-dimensional mapping with a strange attractor." Communications in Mathematical Physics, 50, 69-77.

Iasemidis, L. D. (2003). "Epileptic seizure prediction and control." IEEE Trans Biomed Eng, 50(5), 549-58.

Kalitzin, S. N., Parra, J., Velis, D. N., and Lopes da Silva, F. H. (2007). "Quantification of unidirectional nonlinear associations between multidimensional signals." IEEE Trans Biomed Eng, 54(3), 454-61. 
Ktonas, P. Y., and Mallart, R. (1991). "Estimation of time delay between EEG signals for epileptic focus localization: statistical error considerations." Electroencephalogr Clin Neurophysiol, 78(2), 105-10.

Le Van Quyen, M., Foucher, J., Lachaux, J., Rodriguez, E., Lutz, A., Martinerie, J., and Varela, F. J. (2001). "Comparison of Hilbert transform and wavelet methods for the analysis of neuronal synchrony." $J$ Neurosci Methods, 111(2), 83-98.

Lehnertz, K. (1999). "Non-linear time series analysis of intracranial EEG recordings in patients with epilepsy-an overview." Int J Psychophysiol, 34(1), 45-52.

Lopes da Silva, F., Pijn, J. P., and Boeijinga, P. (1989). "Interdependence of EEG signals: linear vs. nonlinear associations and the significance of time delays and phase shifts." Brain Topogr, 2(1-2), 9-18.

Mars, N., and Lopes da Silva, F. (1983). "Propagation of seizure activity in kindled dogs." Electroencephalography and Clinical Neurophysiology, 56, 194-209.

Mormann, F., Kreuz, T., Rieke, C., Andrzejak, R. G., Kraskov, A., David, P., Elger, C. E., and Lehnertz, K. (2005). "On the predictability of epileptic seizures." Clin Neurophysiol, 116(3), 569-87.

Mormann, F., Lehnertz, K., David, P., and Elger, C. E. (2000). "Mean phase coherence as a measure for phase synchronization and

its application to the EEG of epilepsy patients." Physica. D, 144, 358-369.

Munari, C., Tassi, L., Kahane, P., Francione, S., DiLeo, M., and Quarato, P. (1994). "Analysis of clinical symptomatology during stereo-EEG recorded mesiotemporal lobe seizures." Epileptic seizures and syndromes, W. P, ed., John Libbey \& Co, London.

Pereda, E., DelaCruz, D. M., DeVera, L., and Gonzalez, J. J. (2005). "Comparing Generalized and Phase Synchronization in Cardiovascular and Cardiorespiratory Signals." Biomedical Engineering, IEEE Transactions on, 52(4), 578-583.

Pijn, J. P. (1990). "Quantitative evaluation of EEG signals in epilepsy, nonlinear associations, time delays and nonlinear dynamics," University of Amsterdam, Amsterdam.

Pijn, J. P., and Lopes da silva, F. H. (1993). "Propagation of electrical activity: nonlinear associations and time delays between EEG signals." in Basic Mechanisms of the Eeg, Brain Dynamics, S. Zschocke and E. J. Speckmann, Eds. Boston: Birkhauser, 41-61.

Pikovsky, A., Rosenblum, M., and Kurths, J. (2001). "Synchronization : a universal concept in nonlinear sciences." Cambridge: Cambridge University Press.

Pikovsky, A. S., Rosenblum, M., and Kurths, J. (1996). "Synchronization in a population of globally coupled chaotic oscillators." Europhys. Lett., 34(3), 165-170.

Quian Quiroga, R., Kraskov, A., Kreuz, T., and Grassberger, P. (2002). "Performance of different synchronization measures in real data: a case study on electroencephalographic signals." Phys Rev E Stat Nonlin Soft Matter Phys, 65(4 Pt 1), 041903.

Rosenblum, M., Pikovsky, A., and Kurths, J. (2004). "Synchronization approach to analysis of biological signals." Fluctuation Noise Lett., 4, L53-L62.

Rossler, O. E. (1976). "An equation for continuous chaos." Physics Letters A, 57(5), 397-398.

Schiff, S. J., So, P., Chang, T., Burke, R. E., and Sauer, T. (1996). "Detecting dynamical interdependence and generalized synchrony through mutual prediction in a neural ensemble." Physical Review. E. Statistical Physics, Plasmas, Fluids, and Related Interdisciplinary Topics, 54(6), 6708-6724.

Senhadji, L., Thoraval, L., and Carrault, G. (1996). "Continuous wavelet transform: ECG recognition based on phase and modulus representations and hidden Markov models." Wavelets in medicine and biology, A. Aldroubi and M. Unser, eds., CRC Press, NewYork, 439-463.

Sills, G. J., Leach, J. P., Kilpatrick, W. S., Fraser, C. M., Thompson, G. G., and Brodie, M. J. (2000). "Concentration-effect studies with topiramate on selected enzymes and intermediates of the GABA shunt." Epilepsia, 41 Suppl 1, S30-4.

Stam, C. J., and van Dijk, B. W. (2002). "Synchronization likelihood: an unbiased measure of generalized synchronization in multivariate data sets." Physica D: Nonlinear Phenomena, 163(3-4), 236-251.

Storm van Leeuwen, W., Arntz, A., Spoelstra, P., and Wieneke, G. H. (1976). "The use of computer analysis for diagnosis in routine electroencephalography." Rev Electroencephalogr Neurophysiol Clin, 6(2), 318-27.

Takens, F. (1981). "Lecture Nontes in Mathematics." Springer, 898, 366.

Thatcher, R., Krause, P., and Hrybyk, M. (1986). "Cortico-cortical associations and EEG coherence: a twocompartmental model." Electroenceph Clin Neurophysiol, 64(2), 123-143.

Uhlhaas, P. J., and Singer, W. (2006). "Neural synchrony in brain disorders: relevance for cognitive dysfunctions and pathophysiology." Neuron, 52(1), 155-68.

Wendling, F., Bartolomei, F., Bellanger, J. J., and Chauvel, P. (2001a). "Interpretation of interdependencies in epileptic signals using a macroscopic physiological model of the EEG." Clinical Neurophysiology, 112(7), 1201-1218. 
Wendling, F., Bartolomei, F., Bellanger, J. J., and Chauvel, P. (2001b). "Interpretation of interdependencies in epileptic signals using a macroscopic physiological model of the EEG." Clin Neurophysiol, 112(7), 1201-18.

Wendling, F., Bellanger, J. J., Bartolomei, F., and Chauvel, P. (2000). "Relevance of nonlinear lumpedparameter models in the analysis of depth-EEG epileptic signals." Biol Cybern, 83(4), 367-78. 


\section{Captions}

Table 1: Models of signal generation. Abbreviation SNR denotes “Signal-to-Noise Ratio".

Table 2: Summary of results. For each model (horizontal: M1-M5) and for each studied method (vertical: $\mathrm{R}^{2}$ $\mathrm{SL}$ ), the value of the mean square error (MSE) under null hypothesis (H0, i.e. no coupling), the mean variance (MV) and the median of local relative sensitivity (MLRS) were computed from simulated signals. Values highlighted in grey color correspond to the "best performances', i.e. lowest MSE, lowest MV and highest MLRS. In some cases, methods were found to be insensitive to the variations of the coupling parameter $\mathrm{C}$ in the models. In these cases, the three criteria are not applicable (N/A).

Figure 1: general model proposed to study the behavior of bivariate methods aimed at characterizing the connectivity between coupled systems (unidirectional coupling is tuned using parameter $C$ ) from output signals $X$ and $Y$.

Figure 2: Model of coupled neuronal populations used to generate bivariate time-series data that mimic the temporal dynamics of actual depth-EEG signals (local field potentials).

Figure 3: Results obtained from model M2 in the case coupling parameter $C$ induces a phase relationship only between signals. (a) Example of simulated signals. (b) Estimated relationship (mean value of the estimated quantity $Q(X, Y)$ over all realizations as a function of the coupling degree in the model). (c) Variance of estimation.

Figure 4: Results obtained from the neuronal population model in the case of epileptic EEG activity (sustained spiking activity as observed during ictal periods in epilepsy). (a) Example of simulated signals. (b) Estimated relationship (mean value of the estimated quantity $Q(X, Y)$ over all realizations as a function of the coupling degree in the model). (c) Variance of estimation

Figure 5: A summary of the performances (according to quantitative criteria introduced in this study) of the three families of methods for all considered models of signal generation. For M2(AR), results obtained for phase syn-chronization methods are not represented since these methods were found to be insensitive w.r.t changes of the cou-pling parameter in this case (see table 2).

Figure 6: A "frequency-locking" phenomenon observed at the onset of seizure in a patient with medial temporal lobe epilepsy. a) depth-EEG recordings from medial structures $(\mathrm{X}(\mathrm{t})$ : hippocampus, $\mathrm{Y}(\mathrm{t})$ : entorhinal cortex) performed in a patient with mesial temporal lobe epilepsy. b) Time-frequency representation of the signal energy (spectrogram method computed using the short-term Fourier Transform -STFT- of signals X and Y). White arrows show the occurrence of a fast discharge (around $30 \mathrm{~Hz}$ ) at seizure onset. c) Time- frequency representation of the linear relationship between signals $\mathrm{X}$ and $\mathrm{Y}$. The method consists in the computation of the linear correlation coefficient between signals filtered in narrow sub-bands. Grey arrow shows that the linear correlation increases within the specific frequency sub-band corresponding to the fast activity. d, e) Results obtained from "standard" (i.e. frequency-independent) linear $\left(R^{2}(t)\right)$ and nonlinear $\left(h^{2}(t)\right)$ methods. Black arrows show that the "localized-in-frequency" correlation increase is not detected. 


\begin{tabular}{|c|c|c|c|}
\hline \multirow{3}{*}{$\begin{array}{l}\text { Models of } \\
\text { coupled stochastic } \\
\text { signals }\end{array}$} & M1 & \multicolumn{2}{|r|}{ Generation of broad band signals } \\
\hline & \multirow[t]{2}{*}{ M2 } & $\begin{array}{l}\mathrm{M} 2 \\
(\mathrm{PR})\end{array}$ & $\begin{array}{l}\text { Generation of narrow band signals } \\
\text { (Phase relationship only) }\end{array}$ \\
\hline & & $\begin{array}{l}\mathrm{M} 2 \\
(\mathrm{AR})\end{array}$ & $\begin{array}{l}\text { Generation of narrow band signals } \\
\text { (Amplitude relationship only) }\end{array}$ \\
\hline \multirow{5}{*}{$\begin{array}{l}\text { Models of coupled } \\
\text { nonlinear dynamical } \\
\text { systems }\end{array}$} & M3 & & $\begin{array}{l}\text { Coupled Rössler systems. } \\
\text { Generation of deterministic signals }\end{array}$ \\
\hline & \multirow{4}{*}{ M4 } & $\begin{array}{c}\text { M4a } \\
\left(\begin{array}{c}\text { SNR=inf } \\
\text { ) }\end{array}\right.\end{array}$ & $\begin{array}{l}\text { Coupled Hénon systems: } \\
\text { identical systems. Generation of deterministic } \\
\text { signals }\end{array}$ \\
\hline & & $\begin{array}{c}M 4 a \\
(S N R=2)\end{array}$ & $\begin{array}{l}\text { Coupled Hénon systems: } \\
\text { identical systems. Generation of deterministic } \\
\text { signals + additive noise }\end{array}$ \\
\hline & & $\begin{array}{c}\text { M4b } \\
(\mathrm{SNR}=\mathrm{inf} \\
\text { ) }\end{array}$ & $\begin{array}{l}\text { Coupled Hénon systems: } \\
\text { non-identical systems. Generation of determi- } \\
\text { nistic signals }\end{array}$ \\
\hline & & $\begin{array}{c}M 4 b \\
(S N R=2)\end{array}$ & $\begin{array}{l}\text { Coupled Hénon systems: } \\
\text { non-identical systems. Generation of determi- } \\
\text { nistic signals + additive noise }\end{array}$ \\
\hline \multirow{2}{*}{$\begin{array}{r}\text { Models of coupled } \\
\text { neuronal populations }\end{array}$} & \multirow{2}{*}{ M5 } & $\begin{array}{c}\text { M5 } \\
(B K G)\end{array}$ & $\begin{array}{l}\text { Generation of EEG signals } \\
\text { (background activity) }\end{array}$ \\
\hline & & $\begin{array}{c}\text { M5 } \\
\text { (SPK) }\end{array}$ & $\begin{array}{l}\text { Generation of EEG signals } \\
\text { (Spiking activity) }\end{array}$ \\
\hline
\end{tabular}

Table 1: Models of signal generation. Abbreviation SNR denotes "Signal-to-Noise Ratio". 


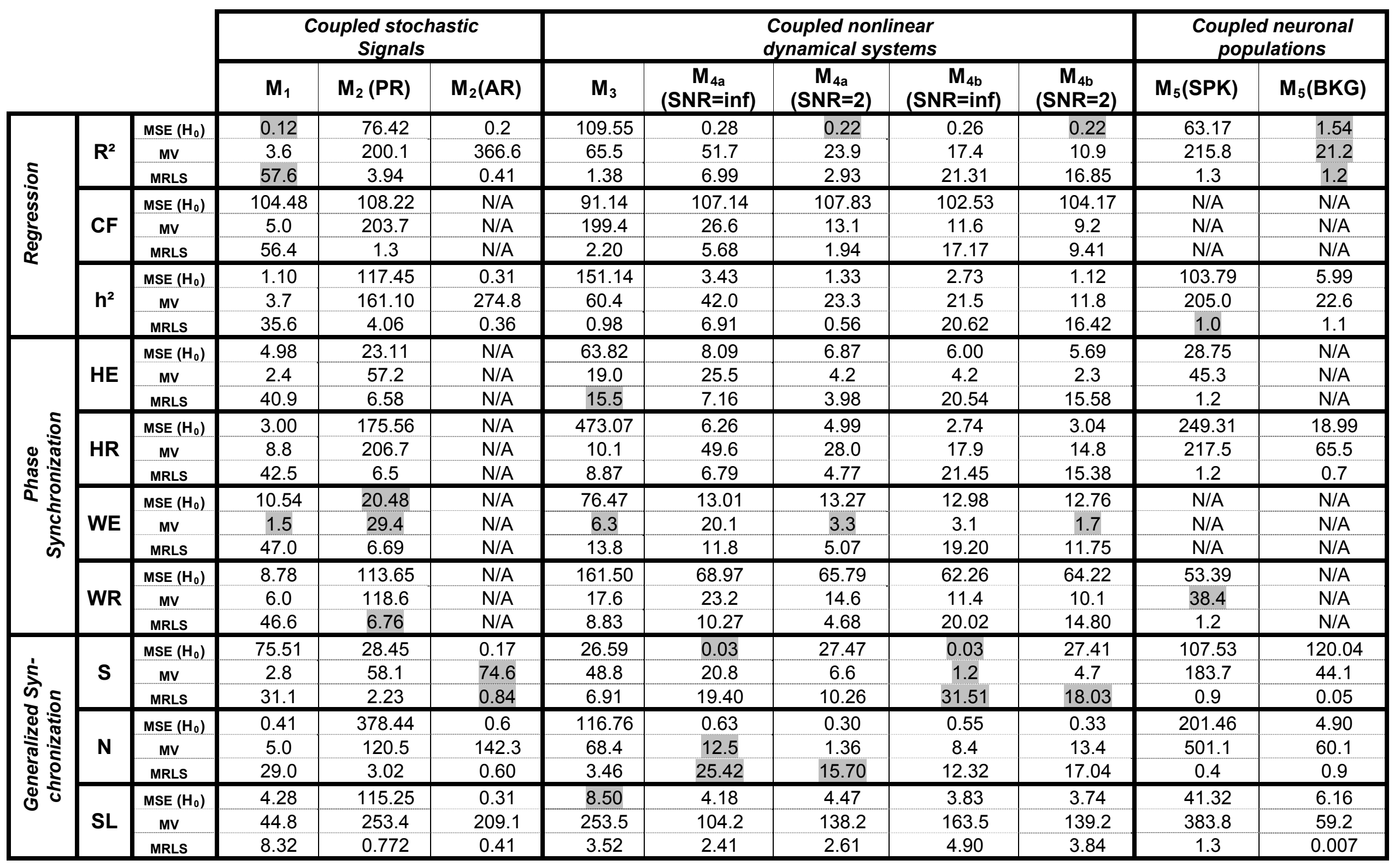





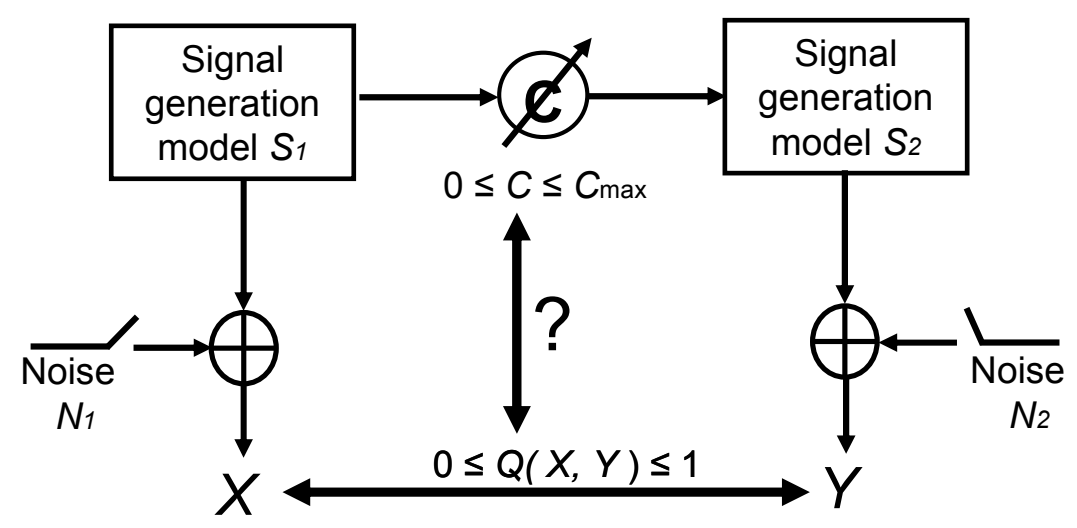

Figure 1: general model proposed to study the behavior of bivariate methods aimed at characterizing the connectivity between coupled systems (unidirectional coupling is tuned using parameter $C$ ) from output signals $X$ and $Y$. 


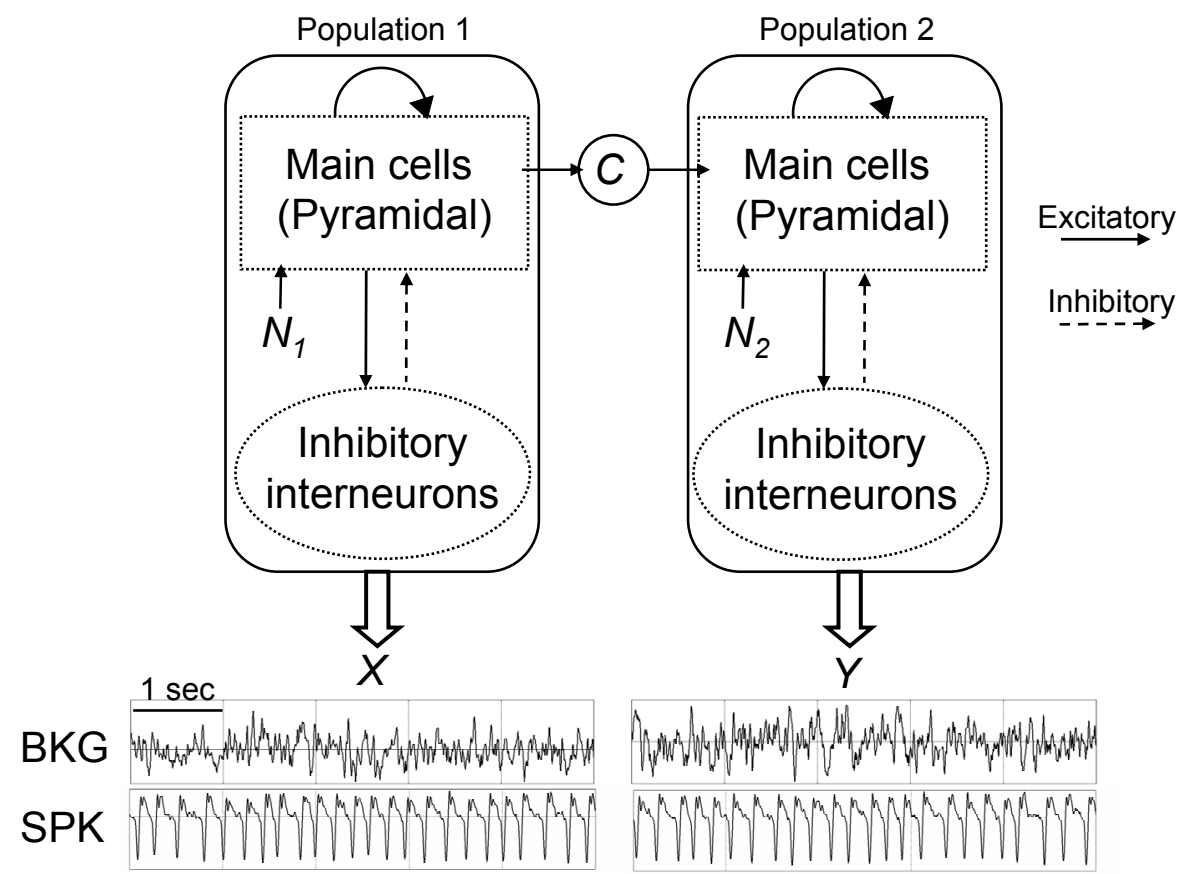

Figure 2: Model of coupled neuronal populations used to generate bivariate time-series data that mimic the temporal dynamics of actual depth-EEG signals (local field potentials). 

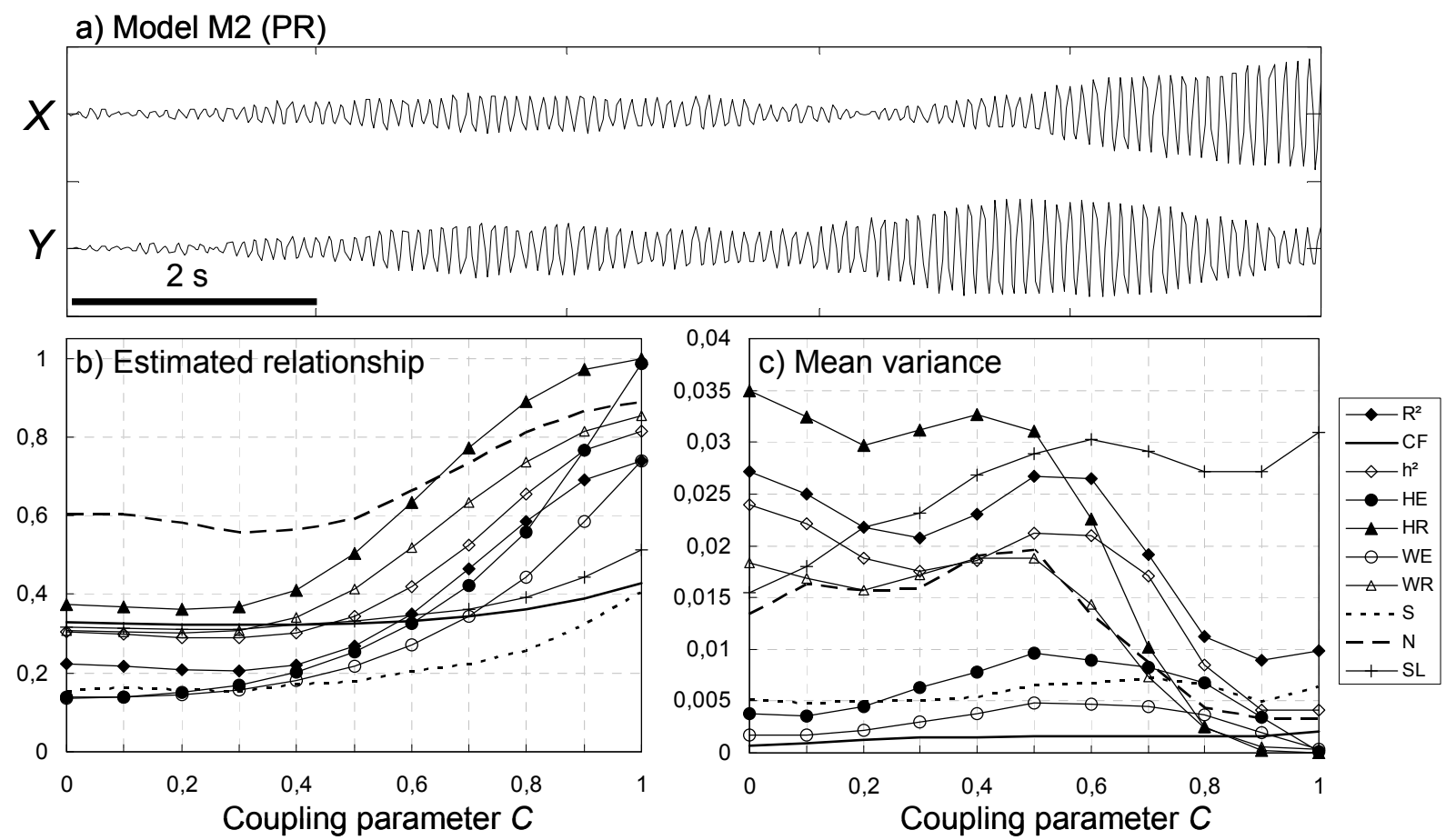

Figure 3: Results obtained from model M2 in the case coupling parameter $C$ induces a phase relationship only between signals. (a) Example of simulated signals. (b) Estimated relationship (mean value of the estimated quantity $Q(X, Y)$ over all realizations as a function of the coupling degree in the model). (c) Variance of estimation. 
a) Model M5 (SPK)
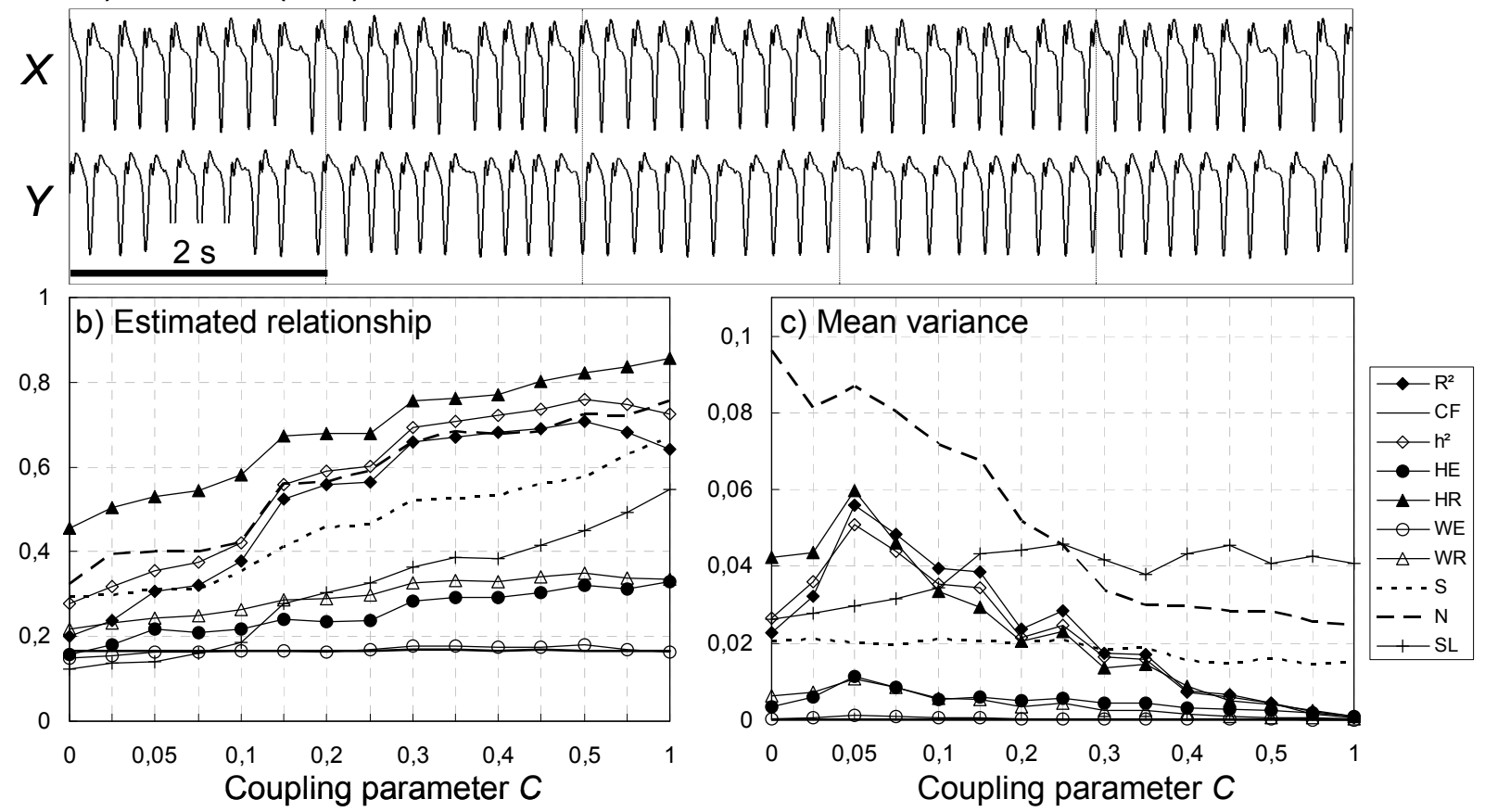

Figure 4: Results obtained from the neuronal population model in the case of epileptic EEG activity (sustained spiking activity as observed during ictal periods in epilepsy). (a) Example of simulated signals. (b) Estimated relationship (mean value of the estimated quantity $Q(X, Y)$ over all realizations as a function of the coupling degree in the model). (c) Variance of estimation 

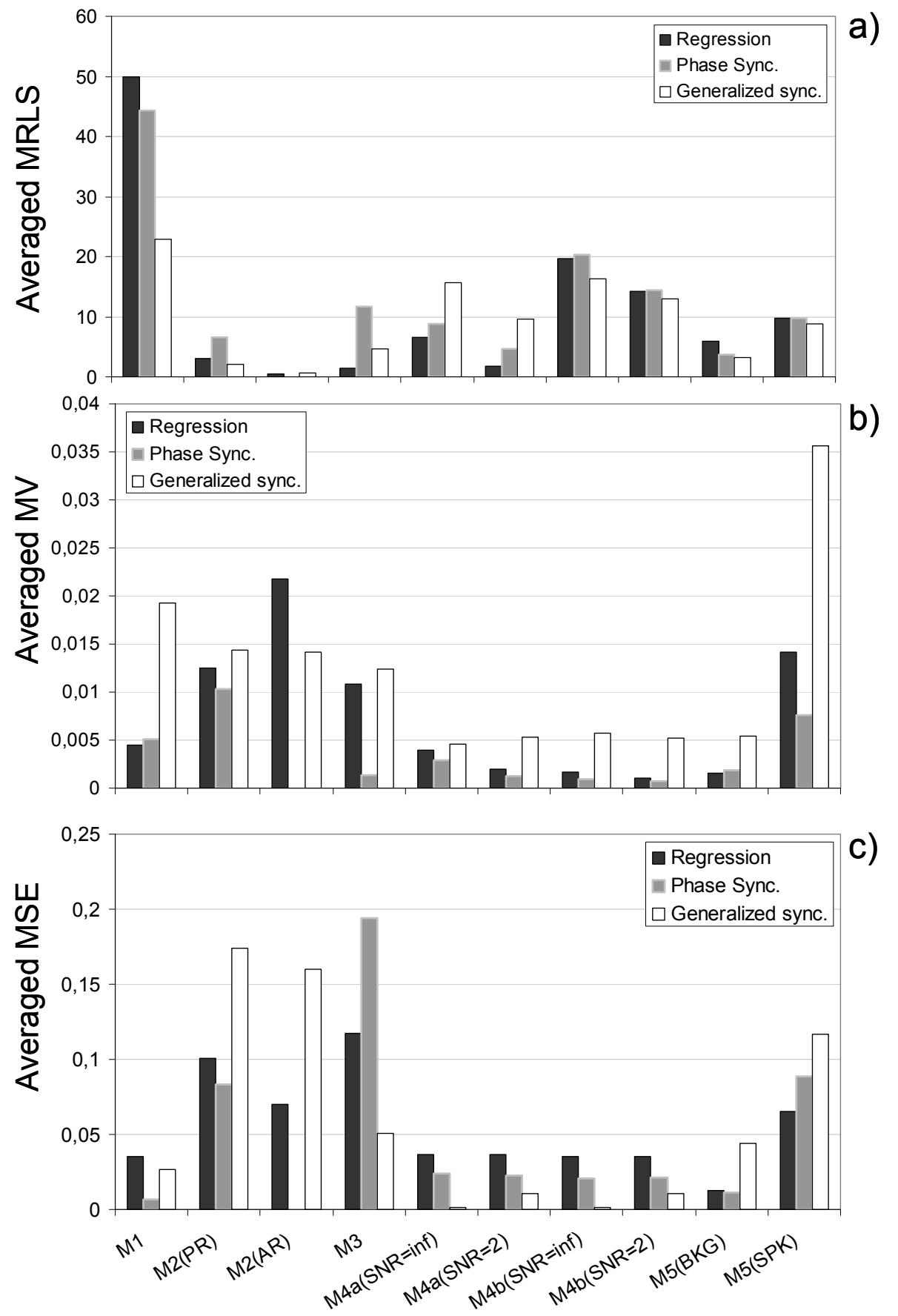

Figure 5: A summary of the performances (according to quantitative criteria introduced in this study) of the three families of methods for all considered models of signal generation. For M2(AR), results obtained for phase synchronization methods are not represented since these methods were found to be insensitive w.r.t changes of the coupling parameter in this case (see table 2). 

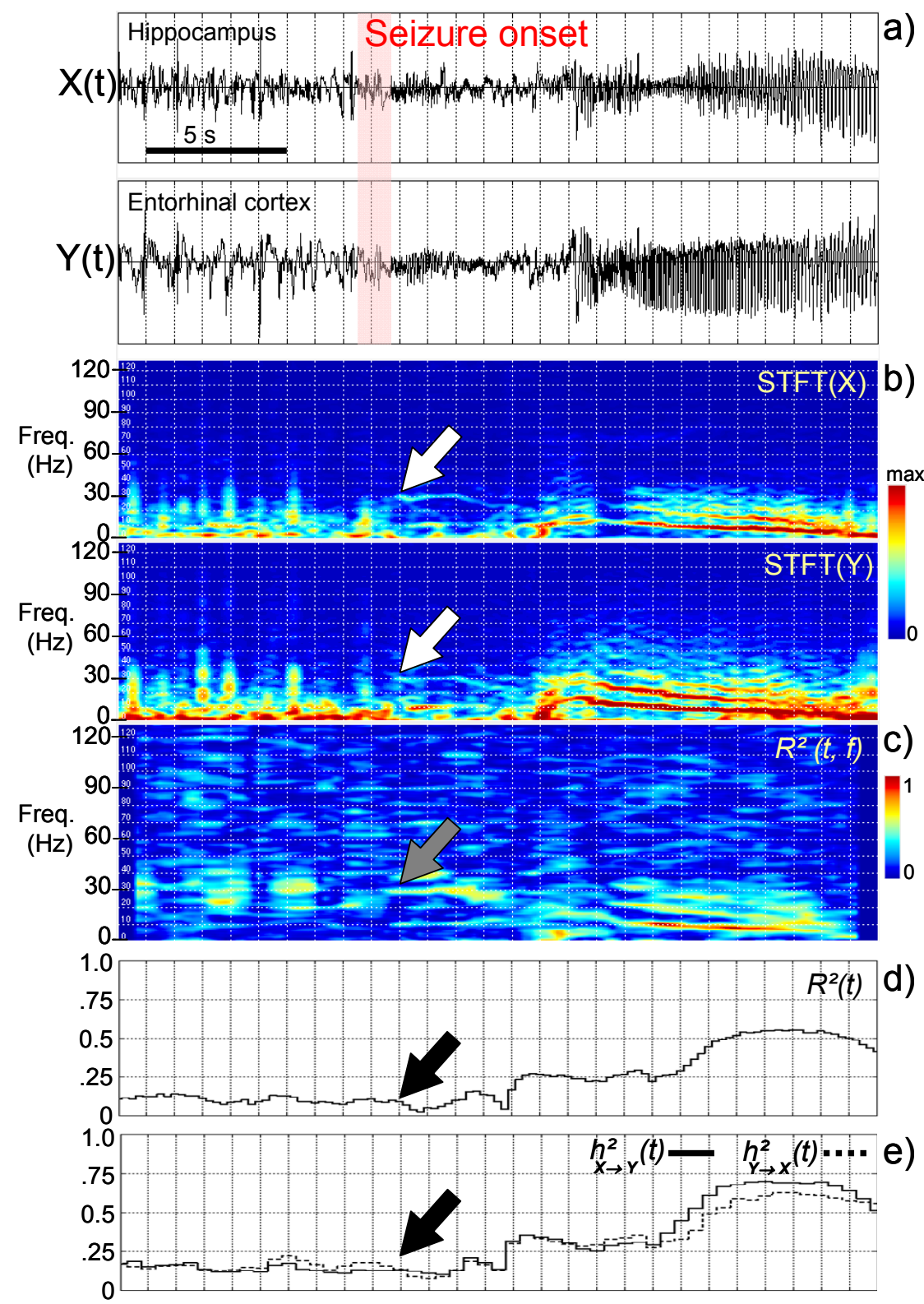

Figure 6: a "frequency-locking" phenomenon observed at the onset of seizure in a patient with medial temporal lobe epilepsy. a) depth-EEG recordings from medial structures $(\mathrm{X}(\mathrm{t})$ : hippocampus, $\mathrm{Y}(\mathrm{t})$ : entorhinal cortex) performed in a patient with mesial temporal lobe epilepsy. b) Time-frequency representation of the signal energy (spectrogram method computed using the short-term Fourier Transform -STFT- of signals X and Y). White arrows show the occurrence of a fast discharge (around $30 \mathrm{~Hz}$ ) at seizure onset. c) Time- frequency representation of the linear relationship between signals $\mathrm{X}$ and $\mathrm{Y}$. The method consists in the computation of the linear correlation coefficient between signals filtered in narrow sub-bands. Grey arrow shows that the linear correlation increases within the specific frequency sub-band corresponding to the fast activity. d, e) Results obtained from "standard" (i.e. frequency-independent) linear $\left(R^{2}(t)\right)$ and nonlinear $\left(h^{2}(t)\right)$ methods. Black arrows show that the "localized-in-frequency" correlation increase is not detected. 